\title{
Review \\ Update on HER-2 as a target for cancer therapy Herceptin in the clinical setting
}

David W Miles

ICRF Breast Cancer Biology Group, Guy's \& St Thomas' Hospital, London, UK

Correspondence: David W Miles, MD, BSc, FRCP, ICRF Breast Cancer Biology Group, 3rd Floor, Thomas Guy House, Guy's Hospital, London SE1 9RT, UK. Tel: +44 (0)20 7955 4542; fax: +44 (0)20 7955 2027; e-mail: d.miles@icrf.icnet.uk

Received: 10 September 2001

Accepted: 21 September 2001

Published: 11 October 2001
Breast Cancer Res 2001, 3:380-384

(C) 2001 BioMed Central Ltd

(Print ISSN 1465-5411; Online ISSN 1465-542X)

\begin{abstract}
Herceptin is the first therapy for breast cancer which targets an oncogene product. This humanized antibody to HER-2 has been shown to have activity as a single agent in a phase II trial of heavily pretreated patients with advanced breast cancer and, in phase III studies, its use with chemotherapy is associated with higher response rates, longer time to progression and improved survival when compared with chemotherapy alone. Retrospective analysis of data from these pivotal trials suggests that attributable benefit of herceptin is greater in those patients who express HER-2 at the highest levels, that is $3+$ expression by immunohistochemistry. Further analysis also implies that cases which are positive for HER-2 by fluorescent in situ hybridization may also benefit from treatment regardless of whether they express HER-2 at the 2+ or 3+ level. Use of herceptin as first-line therapy for metastatic disease in early studies suggest that response rates and clinical benefit rate similar to chemotherapy may be achievable and that survival using this sequential approach may not be compromized. Other combinations of herceptin and chemotherapy have been investigated with phase II data suggesting considerable activity with weekly taxol and when combined with navelbine. The non-linear pharmacokinetics of herceptin suggest that, as doses increased, half-life increases and may be feasible on a 3-weekly schedule. The role of herceptin in the adjuvant setting in the management of breast cancer will be tested in randomized studies of patients who express HER-2 at the highest levels; two of these studies have already begun.
\end{abstract}

Keywords: breast cancer, herceptin, HER-2

\section{Introduction}

Targeted therapies are not a new concept in the field of breast cancer, strategies aimed at targeting the oestrogen receptor having been with us for many years. The identification and exploitation of other targets has taken longer than had been hoped. It is more than 30 years since growth receptors were identified, but only in the past couple of years has a treatment targeting these receptors been available.

Women whose tumours express HER-2 at high levels have a relatively poor prognosis with a median survival of
3 years, compared with 6-7 years for HER-2-negative cases [1]. Many studies published subsequently have demonstrated that HER-2 overexpression is associated with other features of a poor prognosis, namely high tumour grade/S-phase fraction and oestrogen and progesterone receptor negativity [2]. In many series, however, HER-2 status remains an independent poor prognostic feature. Whether HER-2 status is a predictor of response to other treatment modalities in breast cancer, namely hormonal and cytotoxic therapy, remains contentious. Conflicting data are presented in the literature regarding the ability of HER-2 positivity to predict relative resistance to hor-

$\mathrm{Cl}=$ confidence interval; $\mathrm{FISH}=$ fluorescent in situ hybridisation; $\mathrm{IHC}=$ immunohistochemistry. 
monal therapy and chemotherapy. The major difficulties in interpreting these studies is that they are retrospective analyses and, in many instances, there is no satisfactory 'control' arm against which to test attributable benefit of a treatment intervention in different HER-2 subgroups. While debate on this area is bound to continue, it seems unlikely that prospective studies of adjuvant hormonal and/or chemotherapy will be stratified according to HER-2 status. Such is the conflicting nature of the literature regarding HER-2 as a predictive factor that a rational view would be that no active therapeutic option should be disregarded based solely on the HER-2 status of a patient's tumour [3].

\section{Herceptin: pivotal trials}

The two studies that led to the licensing of herceptin as treatment for metastatic breast cancer have now been published [4,5]. A re-analysis of data from both these studies supports the preclinical observation of a relationship between HER-2 expression and growth inhibition of tumour cell lines by antibodies to the receptor (Genentech, San Francisco, data held on file).

In the pivotal phase II study, heavily pretreated patients whose tumour overexpressed HER-2 at the 2 and 3+ levels by immunohistochemistry $(\mathrm{IHC})$ were treated with herceptin as a single agent. Following re-analysis by a 'response evaluation committee', the overall response rate in this group was $15 \%$ with a median survival of 9.1 months [4]. While this response rate itself appears modest, it is noteworthy that the majority of these patients had already received anthracyclines and taxoids, and about one-quarter of patients had received a high dose regimen of chemotherapy. In addition, those patients who did respond to herceptin had a longer duration of response following treatment, compared with their previous regimen of chemotherapy (9.1 months versus 5.2 months). Retrospective analysis of response rate and median survival restricted to the patients whose tumours overexpress HER-2 at the highest levels (immunohistochemical score, $3+$ ) had a response rate of $18 \%$ and a median survival of 16.4 months.

In the pivotal phase III study by Slamon et al. [5], patients were randomised to receive chemotherapy with or without herceptin. Patients were stratified according to whether or not adjuvant chemotherapy contained an anthracycline, such that the majority of patients who did not have adjuvant chemotherapy or whose adjuvant therapy did not contain an anthracycline were randomised to doxorubicin and cyclophosphamide with or without herceptin. In the subgroup of patients who had received an anthracycline in the adjuvant setting, patients were randomised to paclitaxel with or without herceptin.

The principle endpoint of the Slamon et al. study was median time to progression, which for the group as a whole was significantly longer in those patients receiving chemotherapy with herceptin compared with chemotherapy alone (7.4 months versus 4.6 months; $P<0.05)$. Time to progression was significantly longer in each of the chemotherapy subgroups (cyclophosphamide versus herceptin + cyclophosphamide, 6.1 months versus 7.8 months; paclitaxel versus herceptin + paclitaxel, 2.7 months versus 6.9 months). When both chemotherapy subsets were considered, a survival benefit attributable to herceptin with chemotherapy versus chemotherapy alone was noted (median survival, 25 months versus 20 months). This observed survival difference was despite the fact that nearly three-quarters of patients treated initially with chemotherapy alone crossed over to herceptin as a single agent on progression of disease.

It is therefore possible that any observed survival difference attributable to herceptin has been somewhat underestimated. Interestingly, when the benefits of herceptin were analysed retrospectively in those patients expressing HER-2 at the highest level (immunohistochemical score, $3+)$, it became apparent that the difference in most parameters (time to progression, response rate and survival) was greater in those patients whose tumours expressed HER-2 at the $3+$ level compared with the group as a whole. For example, survival in the HER-2 $3+$ subgroup was 29 months for those patients receiving chemotherapy with herceptin, compared with 20 months for those receiving chemotherapy alone [6].

The current limitation of herceptin use in combination chemotherapy remains the cardiac dysfunction observed as part of the pivotal phase III study and when herceptin was combined with anthracycline. Some level of cardiac dysfunction was observed in $27 \%$ of patients treated with doxorubicin/cyclophosphamide with herceptin, compared with only $7 \%$ treated with chemotherapy alone. Cardiac dysfunction reached grade III and IV levels of the New York Heart Association rating in 16\% of patients at some point during therapy, reducing to $6 \%$ of patients once treatment had been completed. The aetiology of the cardiac dysfunction remains unclear, and combinations of herceptin with other anthracyclines, including epirubicin and liposomal doxorubicin, remain the subject of clinical trials. The license for herceptin in combination with chemotherapy is therefore restricted to its use with paclitaxel, where the addition of herceptin increased median survival from 18 to 25 months.

In summary, herceptin is currently licensed for use as a single agent following anthracycline and taxoid chemotherapy or in those patients for whom such therapies are unsuitable, and also in combination with paclitaxel. In both incidences, the current license restricts its use to those patients who tumours overexpress HER-2 at the highest level (3+) as assessed by IHC. 
As well as conventional endpoints, health-related quality of life using the European Organisation for Research and Treatment of Cancer Quality of Life questionnaire QLQC30 was administered at baseline, week 8 and every 3 months thereafter. The five primary prospectively defined quality of life domains examined included global quality of life, physical, social and role functioning as well as fatigue. The use of herceptin with chemotherapy was associated with improvements in all these domains when compared with chemotherapy alone [7].

\section{Testing of samples for HER-2 status}

Debate continues regarding the best way of testing tissue samples for the presence of high levels of HER-2 receptor. The vast majority of studies examining the use of HER2 overexpression as a prognostic and predictive factor have been carried out using IHC. This is obviously a wellestablished and easy to use technique that is widely available. Part of the problem encountered in interpreting the prognostic and predictive data from HER-2 is also an issue for testing the suitability of patients for herceptin; $\mathrm{IHC}$ analysis may be subject to the vagaries of differences in tissue fixation. In addition, the use of different antibodies, other methodologies and scoring systems may render this technique less than completely objective.

Fluorescent in situ hybridisation (FISH) detects HER-2 at the DNA level and is specific and very sensitive. As a technique, however, it has limited availability, it is more expensive than $\mathrm{IHC}$ and it requires specialised equipment. Comparisons between IHC analysis and analysis by FISH of many of the samples from the phase II and phase III pivotal studies show a good concordance between the two techniques. Nevertheless, from the data presented by Mass et al. [6], it is clear that $11 \%$ of cases that were 3+ by $\mathrm{IHC}$ are actually $\mathrm{FISH}$-negative and, conversely, a significant proportion (24\%) of cases that are $2+$ by $\mathrm{IHC}$ are FISH-positive. Given the inherent variability of testing by $\mathrm{IHC}$, it is a concern that cases that are FISH-positive but only $2+$ by $\mathrm{IHC}$ might indeed benefit from herceptin, although this has not been evaluated prospectively. Nevertheless, it is worthy of note that, for the monotherapy study, the objective response rate in the $\mathrm{FISH}$-positive group is $21 \%$ and $18 \%$ in the patients whose tumours were $3+$ by IHC. Time to progression in these two groups $(\mathrm{IHC} 3+$ and FISH-positive) were identical at 3.2 months. Similarly, in the pivotal phase III study, response rates, time to progression and survival were similar in those patients who were FISHpositive compared with the group whose tumours were $\mathrm{IHC} 3+$. On these grounds, it has been suggested that although patients whose tumours express HER-2 at the 3+ level are those most likely to benefit from herceptin, patients whose tumours express HER-2 at the 2+ level but who are FISH-positive should also be considered for treatment with herceptin. It is expected that a license variation will be applied for to account for this group of patients.

\section{Future development of herceptin First line monotherapy}

Herceptin has been tested in the first line treatment of metastatic breast cancer in patients who were unsuitable for or who declined chemotherapy. Vogel et al. performed a randomised phase II study comparing two different dose schedules of herceptin [8]. A total of 114 HER-2positive patients were randomised to receive herceptin at standard doses $(4 \mathrm{mg} / \mathrm{kg}$ loading dose followed by $2 \mathrm{mg} / \mathrm{kg}$ weekly) or high dose ( $8 \mathrm{mg} / \mathrm{kg}$ loading dose followed by $4 \mathrm{mg} / \mathrm{kg}$ weekly). Response rates in the two groups were similar. When combined, the overall response rate (complete plus partial responses) for the group as a whole was 26\% (95\% confidence interval [Cl], 18-34\%). In those patients who overexpressed HER-2 at the $3+$ level by IHC, the response rate was noted to be $35 \%(95 \% \mathrm{Cl}, 24-44 \%)$. If disease stabilisation for longer than 6 months was added to the complete and partial responders to define a clinical benefit rate, then the clinical benefit rate in the patients whose tumours expressed HER-2 at the IHC $3+$ level was $48 \%$. It is noteworthy that no complete or partial responses were noted in patients whose tumours expressed HER-2 at the IHC 2+ level. If only patients whose tumours expressed HER-2 by FISH were considered, then the overall response rate (complete and partial response) was $41 \%(95 \% \mathrm{Cl}, 26-56 \%)$.

The $95 \%$ Cls on these response rate estimates, taking all comers, IHC $3+$ or FISH-positive patients, all overlap. There is, however, a trend towards higher response rate in those patients who are perhaps better defined by FISH. Cross-trial comparisons between Vogel et al.'s study [8] and the pivotal phase III study [5] suggest that the use of herceptin as monotherapy in FISH-positive patients rather than in combination may not ultimately compromise outcome. The median survival in 41 patients who were FISH-positive receiving herceptin as first line monotherapy was 23 months, compared with a median survival of 26.8 months in a 125 patients from the pivotal study who had herceptin in combination with chemotherapy. While logistically difficult, a sequential versus combination study would be informative in this respect.

\section{Herceptin in combination with other agents}

The combination of herceptin and navelbine has been tested in the phase II setting [9]. The overall response rate to the combination in patients with metastatic disease was $75 \%$, and when considering patients whose tumours overexpressed HER-2 at the IHC 3+ level the overall response rate was $80 \%$. The combination was well tolerated and clearly warrants further investigation. Given the weekly schedule of herceptin and the observations that weekly paclitaxel has relatively high activity, the combination of herceptin and weekly paclitaxel was a logical one to pursue. 
Seidman et al. examined the use of herceptin and paclitaxel in patients with metastatic disease irrespective of HER-2 status [10]. The original intention of the study was to compare roughly equal numbers of HER-2-positive and HER-2-negative patients and to make comparisons of response rate according to different assay techniques. Overall, the combination was associated with response rates of $80 \%$ in patients who were HER2-positive and only $43 \%$ in patients who were HER-2negative. Response was probably better defined by the use of the monoclonal antibody, TAB 250, rather than the now more widely used HercepTest kit. Given the relative lack of efficacy of herceptin in HER-2-negative patients, it seems unlikely that this will be the subject of much further study. Whether the weekly schedule of paclitaxel in combination with herceptin is superior to the 3-weekly schedule in those patients whose tumours overexpress HER-2 will clearly need to be tested in the context of a randomised study.

Given the observed cardiotoxicity in the pivotal phase III trial, current combinations of herceptin and anthracyclines are in the minority. Some groups are, however, investigating the possible use of the slightly less cardiotoxic epirubicin as well as liposomal doxorubicin. A current randomised phase II study is examining the contribution of herceptin to docetaxel as first line therapy for metastatic disease. Preclinical data suggest that one of the more potent 'synergistic' combinations of herceptin would be with platinum and a taxoid. Studies by Slamon et al. have examined this combination [11].

\section{Herceptin dose scheduling}

Increasing the dose interval of herceptin would obviously be more convenient, would increase patient compliance and would render more feasible studies of herceptin in the adjuvant setting. Pharmacokinetic modelling suggest that a 3-weekly administration may be feasible. This is largely on the basis that herceptin demonstrates dose-dependent non-linear pharmacokinetics with faster clearance and shorter half-life at doses less than $100 \mathrm{mg}$. More recent data from Gelmon et al. assess the toxicity and safety of 3-weekly herceptin and paclitaxel [12]. Herceptin was given at a loading dose of $8 \mathrm{mg} / \mathrm{kg}$ with subsequent doses of $6 \mathrm{mg} / \mathrm{kg}$ every 3 weeks. Overall, the half-life of herceptin in this study was in the order of 21 days. Biological relevant trough levels were attained in a similar time period to the schedule of weekly administration. A similar profile of toxicity in reduction in left ventricular ejection fraction was noted as for weekly administration. Herceptin given every 3 weeks is clearly a feasible schedule. It is noteworthy, however, that with a half-life of 21 days, total clearance of herceptin could take up to 18 weeks, which might have implications for anthracycline regimens following discontinuation of herceptin.

\section{Herceptin in the adjuvant setting}

HER-2 amplification and overexpression is clearly an early event in the pathogenesis of breast cancer. Indeed, overexpression in ductal carcinoma in situ is significantly higher than in invasive disease (reviewed in [2]). With the observation of the activity of herceptin as single agent therapy and considering its capacity to improve survival in metastatic disease when used in combination with chemotherapy, the development of adjuvant studies was clearly logical.

The NSABP B31 study compares paclitaxel with paclitaxel and concurrent herceptin following four cycles of cyclophosphamide. Eligible patients are those with tumours overexpressing HER-2 at the $3+$ level by IHC or those who are FISH-positive. It is expected that 2700 patients will accrue to this study. The intergroup trial N9831 is similar to NSABP B31, but as a three-arm study that compared paclitaxel alone with paclitaxel and concurrent herceptin versus paclitaxel followed by herceptin. Again, eligible patients are those who express HER-2 at the highest levels by IHC or FISH. One thousand patients per arm will be accrued over 4.5 years. In both these studies, cardiac safety is being monitored carefully with safety analyses after predetermined levels of accrual.

The BCIRG trial of herceptin in the adjuvant setting is also a three-arm study, which tests the use of docetaxel following four courses of cyclophosphamide with docetaxel and herceptin given on a weekly schedule for 1 year. The third arm of this study aims to compare these 'standard' anthracycline style regimens with docetaxel and cisplatin or carboplatin times six with weekly herceptin for 1 year. The herceptin adjuvant trial is a slightly more pragmatic study in which eligible patients (tumour HER-2 3+ or FISH-positive) complete primary management with chemotherapy and possibly radiotherapy, and are stratified by type of chemotherapy to one of three arms: mainly observation, herceptin given on a 3-weekly schedule for 12 months, and herceptin given on a 3-weekly schedule for 24 months. This is the only current randomised adjuvant trial proposed that uses the 3-weekly schedule and is also testing two durations of herceptin. Again, cardiac function is being monitored closely.

\section{Conclusions}

HER-2 testing should be considered in patients with breast cancer, based not only on its potential usefulness as a prognostic or predictive factor, but more to define whether the humanised monoclonal antibody to this growth factor receptor may be of use in patient management. The use of herceptin in combination with chemotherapy has been demonstrated to prolong survival in women with metastatic breast cancer. Sadly, very few agents have demonstrated such a benefit in this setting and, certainly, the additional toxicity associated with this 
benefit seems small. New combinations and schedules using herceptin seem very promising, but will need to be tested further. The role of this agent and the adjuvant treatment of breast cancer will be tested in the adjuvant studies currently ongoing.

\section{References}

1. Slamon DJ, Clark GM, Wong SG, Levin WJ, Ullich A, McGuire WL: Human breast cancer: correlation of relapse and survival with amplification of the HER-2/neu oncogene. Science 1987, 235:177-182.

2. Ross JS, Fletcher JA: HER-2/neu (c-erb-B2) gene and protein in breast cancer. Am J Clin Pathol 1999, 112(suppl 1):S53S67.

3. Yamauchi $H$, Stearns V, Hayes DF: When is a tumour marker ready for prime time? A case study of c-erbB-2 as a predictive factor in breast cancer. J Clin Oncol 2001, 19:2334-2356.

4. Cobleigh MA, Vogel CL, Tripathy D, Robert NJ, Scholl S, Fehrenbacher L, Wolter JM, Paton V, Shak S, Lieberman G, Slamon DJ: Multinational study of the efficacy and safety of humanised anti-HER2 monoclonal antibody in women who have HER2overexpressing metastatic breast cancer that has progressed after chemotherapy for metastatic disease. J Clin Oncol 1999, 17:2639-2648.

5. Slamon DJ, Leyland-Jones B, Shak S, Fuchs H, Paton V, Bejamonde A, Fleming T, Eiermann W, Wolter J, Pegram M, Beselga J, Norton L: Use of chemotherapy plus a monoclonal antibody against HER2 for metastatic breast cancer that overexpress HER2. N Engl J Med 2001, 344:783-792.

6. Mass RD, Sanders C, Charlene K, Johnson L, Everett T, Anderson $\mathrm{S}$ : The concordance between the clinical trials assay (CTA) and fluorescence in situ hybridisation (FISH) in the herceptin pivotal trials [abstract 291]. Proc Am Soc Clin Oncol 2001, 20: $75 \mathrm{a}$.

7. Osoba $D$, Burchmore M: Health-related quality of life in women with metastatic breast cancer treated with trastuzumab (herceptin). Semin Oncol 1999, 26(4 suppl 12):84-88.

8. Vogel C, Cobleigh M, Tripathy D, Harris L, Fehrenbacher L, Slamon D, Ash M, Novotny W, Stewart S, Shak S: First-line, nonhormonal, treatment of women with HER2 overexpressing metastatic breast cancer with herceptin (trastuzumab, humanised anti-HER2 antibody) [abstract 275]. Proc Am Soc Clin Oncol 2001, 20:71a.

9. Burstein HJ, Juter I, Campos SM, Gelman RS, Tribou L, Parker LM, Manola J, Younger J, Matulouis U, Bunnell CA, Partridge AH, Richardson PG, Clarke K, Shulman LN, Winer EP: Clinical activity of trastuzumab and vinorelbine in women with HER2-overexpressing metastatic breast cancer. J Clin Oncol 2001, 19: 2722-2730.

10. Seidman AD, Fornier MN, Esteva FJ, Tan L, Kaptain S, Bach A, Panageas KS, Arroyo C, Valero V, Currie V, Gilewski T, Theodoulou M, Moynahan ME, Moasser M, Sklarin N, Dickler M, D'Andrea G, Cristofanilli M, Rivera E, Hortobagyi GN, Norton L, Hudis CA: Weekly trastuzumab and paclitaxel therapy for metastatic breast cancer with analysis of efficacy by HER2 immunophenotype and gene amplification. J Clin Oncol 2001, 19:2587-2595

11. Slamon DJ, Patel R, Northfelt R, Pegram M, Rubin J, Sebastian G, Tannenbaum S, Sanchez J, Quan E, Toppmeyer D, Overmoyer B, Nabholtz J: Phase II pilot study of herceptin combined with taxotere and carboplatin $(\mathrm{TCH})$ in metastatic breast cancer (MBC) patients overexpressing the HER2-neu proto-oncogene: a pilot study of the UCLA network [abstract 193]. Proc Am Soc Clin Oncol 2001, 20:49a.

12. Gelmon K, Arnold A, Verma S, Ayoub J, Hemmings F, LeylandJones B: Pharmacokinetics (PK) and safety of tastuzumab (Herceptin ${ }^{\circledR}$ ) when administered every three weeks to women with metastatic breast cancer [abstract 271]. Proc Am Soc Clin Oncol 2001, 20:69a. 